\title{
Molecular Weight Dependence of Critical Amplitudes in Polymer Solutions
}

\author{
Kenji SHINOZAKI and Takuhei NosE \\ Department of Polymer Chemistry, Tokyo Institute of Technology, \\ Ookayama, Meguro-ku, Tokyo 152, Japan.
}

(Received June 30, 1981)

\begin{abstract}
Universal ratios of critical amplitudes give relations among the exponents characterizing molecular weight dependence of critical amplitudes in polymer solutions. These relations can be deduced from special scaling forms of the free energy density and correlation function proposed. The classical values of the exponents are also derived from the Flory-Huggins theory.
\end{abstract}

KEY WORDS Critical Phenomena / Critical Amplitude / Scaling Theory /

Studies on critical phenomena in polymer solutions have had a rather long history since Debye ${ }^{1}$ proposed a theory for critical opalescence in a polymer solution. Recently the theory of critical phenomena has undergone considerable development. Universality is the main concept in the recent theory; that is, the values of the critical exponents are independent of any microscopic details of a system if the system belongs to the same symmetry class. Recent experimental investigations in polymer solutions are thus concerned mainly with critical exponents, especially $\beta$, which is the exponent characterizing coexistence curves, and the universal values of the critical exponent has been obtained.

On the other hand, critical amplitudes depend on microscopic details of a system. Since, in a polymer solution, the molecular weight dependence of various quantities is always of interest, attention should be focused on that of critical amplitudes. Experimental investigations in this regard have appeared recently. ${ }^{5,6}$ With regard to correlation length, its molecular weight dependence has been the subject of inquiry since the proposal of the Debye theory. ${ }^{1}$ Other critical amplitudes have not however been sufficiently investigated experimentally or theoretically.

Although the values of critical amplitudes vary according to the system, there are universal ratios among critical amplitudes. ${ }^{7-9}$ These ratios directly result from the universality of critical phenomena.
Universality in this form is called two-scale-factor universality, meaning that the material dependence of critical amplitudes can be scaled by two materialdependent factors. This provides us with a very useful theoretical means for considering the molecular weight dependence of critical amplitudes in polymer solutions. Along this line, the present study is theoretically concerned with the molecular weight dependence of critical amplitudes. In the first section, we derive the exponents characterizing the molecular weight dependence of critical amplitudes from the Flory-Huggins theory. General relations among the exponents, which we have reported recently, ${ }^{10}$ are described with detailed derivations in the second section. Finally, a comparison is made with experiments and other theoretical investigations.

\section{CLASSICAL TREATMENT}

Consider the Flory-Huggins free energy $F$ near the critical point. Expanding the free energy about the critical point up to the quartic term of the concentration and inserting the critical conditions, we obtain,

$$
F-F_{\mathrm{c}}=\partial^{3} F /\left.\partial T \partial \phi^{2}\right|_{\mathrm{c}} \Delta T \Delta \phi^{2}+\partial^{4} F /\left.\partial \phi^{4}\right|_{\mathrm{c}} \Delta \phi^{4}
$$

where $\phi$ is the polymer concentration in the volume fraction, $\Delta \phi \equiv \phi-\phi_{\mathrm{c}}, \Delta T \equiv T-T_{\mathrm{c}}$, and the subscript $\mathrm{c}$ denotes the value at the critical point. Other 
terms such as those that are linear in $\Delta \phi$ or $\Delta T$ in the expansion have no effect on critical properties as can be easily confirmed; thus, these terms have been omitted. Equation 1 has the same form as the Ginsburg-Landau form for free energy, ${ }^{11}$ where $\Delta \phi$ plays a role of an order parameter. For a sufficiently large molecular weight, the two derivatives in eq 1 depend on $N$ (molecular weight or degree of polymerization) as $\partial^{3} F / \partial T \partial \phi^{2} \sim N^{0}$ and $\partial^{4} F / \partial \phi^{4} \sim N^{1 / 2}$. Hence, we rewrite eq 1 , omitting the coefficients and scaling by $T_{\mathrm{c}}$ from a dimensional consideration (where the Boltzmann constant is omitted for simplicity), as,

$$
F_{\mathrm{s}} / T_{\mathrm{c}} \simeq \tau \Delta \phi^{2}+N^{1 / 2} \Delta \phi^{4}
$$

where $\tau \equiv \Delta T / T_{\text {c }}$.

Following Landau's argument, ${ }^{11}$ we can easily deduce the following molecular weight dependence of critical amplitudes.

Minimizing $F_{\mathrm{s}}$ with respect to $\Delta \phi$, we obtain,

$$
\begin{aligned}
\Delta \phi^{2} & =0 & & \tau>0 \\
& \simeq|\tau| / N^{1 / 2} & & \tau<0
\end{aligned}
$$

which indicates that the critical amplitude of the coexistence curve is proportional to $N^{-1 / 4}$.

For the free energy, eq 3 yields,

$$
\begin{aligned}
F_{\mathrm{s}} / T_{\mathrm{c}} & =0 & & \tau>0 \\
& \simeq N^{-1 / 2} \tau^{2} & & \tau<0
\end{aligned}
$$

which gives,

$$
\Delta C \propto N^{-1 / 2}
$$

where $\Delta C$ is the discontinuity in the specific heat at the critical point.

As far as the $N$ dependence is concerned, the osmotic pressure of a polymer, $\pi$ (see eq 14), may be identified with the derivative $\partial F / \partial \phi$. Therefore, differentiating eq 2 with respect to $\Delta \phi$, we obtain an equation-of-state:

$$
\partial\left(F_{\mathrm{s}} / T_{\mathrm{c}}\right) / \partial \phi \sim p / T_{\mathrm{c}} \simeq N^{1 / 2} \Delta \phi^{3}+\tau \Delta \phi
$$

where $p \equiv \pi-\pi_{\mathrm{c}}$. At the critical temperature,

$$
p / T_{\mathrm{c}} \simeq N^{1 / 2} \Delta \phi^{3}
$$

Isothermal osmotic compressibility $\chi$ is defined as,

$$
\chi \equiv-\left(1 / \phi_{\mathrm{c}}\right)(\partial \Delta \phi / \partial p) \equiv-\left(1 / \phi_{0 \mathrm{c}}\right)\left(\partial \Delta \phi_{0} / \partial p_{0}\right)
$$

where $p_{0}$ and $\phi_{0}$ denote the quantities of the solvent.
Differentiation of eq 5 with respect to $\Delta \phi$ yields,

$$
\begin{aligned}
\left(\phi_{\mathrm{c}} \chi\right)^{-1} & \simeq \tau & & \tau>0 \\
& \simeq N^{1 / 2}|\tau| / N^{1 / 2}+|\tau| \simeq|\tau| & & \tau<0
\end{aligned}
$$

Thus, $\phi_{\mathrm{c}} \chi$ is independent of $N$ and then, since $\phi_{\mathrm{c}} \propto N^{-1 / 2}$,

$$
\chi \simeq N^{1 / 2}|\tau|^{-1} \quad \tau \gtrless 0
$$

To derive the molecular weight dependence of the correlation length $\xi$ and the wave-vector-dependent osmotic compressibility $\chi(k)$ ( $k$ : wave vector), we must formulate a local free energy density $f_{\mathrm{s}}(\vec{x})$ and hence the gradient term of the free energy which is written as $\kappa\{\nabla \Delta \phi(\vec{x})\}^{2}$. Following the proposition by Vrij, ${ }^{12}$ which is an improvement of the Debye theory, ${ }^{1}$ or an interfacial tension theory by Nose, ${ }^{13}$ we put $\kappa \propto N^{1 / 2}$; i.e., the square-gradient term is not proportional to $N$ as was proposed by Debye. ${ }^{1} \mathrm{We}$ assume furthermore that the form of eq 1 or 2 holds locally. Then, the local free energy density $f_{\mathrm{s}}(\vec{x})$ has the form:

$$
f_{\mathrm{s}}(\vec{x}) \simeq N^{1 / 2}\{\nabla \Delta \phi(\vec{x})\}^{2}+\Delta T \Delta \phi(\vec{x})^{2}+N^{1 / 2} \Delta \phi(\vec{x})^{4}
$$

Omitting the $\Delta \phi(\vec{x})^{4}$ term, as is usually done in deriving classical exponents, and transforming to the Fourier space, we can calculate the correlation function $\hat{C}(k)$ by the Gaussian integral to obtain

$$
\hat{C}(k) \simeq 1 /\left(N^{1 / 2} k^{2}+\tau\right) \propto 1 /\left(k^{2} \xi^{2}+1\right)
$$

which yields,

$$
\xi \simeq N^{1 / 4} \tau^{-1 / 2}
$$

and,

$$
\left.\chi(k) \equiv \hat{C}(k)\right|_{\tau=0} \simeq N^{-1 / 2} k^{-2}
$$

Interfacial tension near the critical point was derived by Nose $^{13}$ and by Vrij and Roeberson ${ }^{14}$ who used the Cahn-Hilliard theory of interfacial tension and obtained,

$$
\sigma \simeq N^{-1 / 4}|\tau|^{3 / 2}
$$

Thus, the molecular weight dependence of the main critical amplitudes have been derived within classical treatment.

\section{SCALING LAWS OF $N$ DEPENDENCE}

Classical theory predicts that critical amplitudes 
vary in some powers of $N$ for sufficiently large $N$. Experiments $^{5,6,15}$ also show such $N$ dependence, although the values of the exponents are somewhat different from the classical ones. Therefore, we may put the following $N$ dependence of the critical amplitudes.

$$
\begin{aligned}
& \xi=\xi_{0}|\tau|^{-v} \quad \propto N^{n}|\tau|^{-v} \\
& \chi=\Gamma|\tau|^{-\gamma} \quad \propto N^{g}|\tau|^{-\gamma} \\
& C=\left.A|\alpha| \tau\right|^{-\alpha} \quad \propto N^{c}|\tau|^{-\alpha} \\
& \Delta \phi=B|\tau|^{\beta} \quad \propto N^{b}|\tau|^{\beta} \\
& \sigma=\sigma_{0}|\tau|^{(d-1) v} \propto N^{m}|\tau|^{(d-1) v} \quad(\tau<0, p=0) \\
& p=D \Delta \phi^{\delta} \quad \propto N^{j} \Delta \phi^{\delta} \quad(\tau=0, p \neq 0) \\
& \chi(k)=\hat{D} k^{\eta-2} \quad \propto N^{k} k^{\eta-2} \quad(\tau=0, p=0)
\end{aligned}
$$

where we have used conventional notation ${ }^{7-9}$ for the critical amplitudes. We have omitted in $\xi, \chi$ and $C$, the differences between the critical amplitudes for $\tau>0$ and for $\tau<0$, since the ratios of $\xi_{0}^{+} / \xi_{0}^{-}, \Gamma^{+} / \Gamma^{-}$, and $A^{+} / A^{-}$, where + correspond to $\tau>0$ and - to $\tau<0$, are universal constants. Therefore, the $N$ dependences are the same for $\tau \gtrless 0$. Also, we need to put the $N$ dependences of $\phi_{\mathrm{c}}$ and $T_{\mathrm{c}}$ as,

$$
\phi_{\mathrm{c}} \propto N^{r} \quad \text { and } \quad T_{\mathrm{c}} \propto N^{s}
$$

For instance, $s=0$ and $r=-1 / 2$ for a polymersolvent system and $s=1$ and $r=0$ for a polymerpolymer system according to the Flory-Huggins theory.

The concept of the two-scale-factor universality, which results directly from the postulate that the only two of the critical exponents are independent, affirms that the material-dependence of the critical amplitudes enters through only two scale factors. Thus, among the critical amplitudes, there are material-independent relations called the universal ratios. Some of these are listed in Table I. As far as the molecular weight dependence is concerned, we can construct special scaling forms of a free energy density and a correlation function from which the scaling laws, including the $N$ dependence of the critical amplitudes, can be derived.

Consider a thermodynamic potential $G$ corresponding to the semi-grand canonical ensemble:

$$
G=F-N_{1} \mu_{1}
$$

where $F$ is the Helmholtz free energy, $N_{1}$ the number of polymer molecules, and $\mu_{1}$ the chemical potential of the polymer. Thus,

$$
\partial G / \partial \mu_{1}=-N_{1}
$$

The osmotic pressure $\pi$ of the polymer is related to $\mu_{1}$ by

$$
\pi=-\left(\mu_{1}-\mu_{1}^{0}\right) / v_{1}
$$

where $\mu_{1}{ }^{0}$ is the chemical potential of the pure polymer and $v_{1}$ the volume of the polymer. From eq 13 we have,

$$
\partial G / \partial \pi \simeq v_{1} N_{1} \simeq V \phi_{1}
$$

where $V$ is the volume of the system and $\phi_{1}$ is the volume fraction of the polymer. Critical properties are described by the singular part of the thermodynamic potential, $G_{\mathrm{s}}$, the osmotic pressure $p$ $\left(\equiv \pi-\pi_{\mathrm{c}}\right)$, and the temperature $\tau\left[\equiv\left(T-T_{\mathrm{c}}\right) / T_{\mathrm{c}}\right]$. We obtain, corresponding to eq 15 ,

$$
\begin{aligned}
\partial G_{\mathrm{s}} / \partial p & =v_{1}\left(N_{1}-N_{1 \mathrm{c}}\right) \\
& =v_{1}\left(\partial N_{1} / \partial \phi_{1}\right)_{N_{0}} \Delta \phi_{1} \\
& \simeq V \Delta \phi_{1} / \phi_{0}
\end{aligned}
$$

where $\Delta \phi_{1} \equiv \phi_{1}-\phi_{\mathrm{c}}$ and $\phi_{0}$ is the volume fraction of the solvent. Equation 16 shows that $\Delta \phi_{1}$ is the conjugate variable of $p$. Thus, the scaling form of $g_{\mathrm{s}}$, the density of the singular part of the thermodynamic potential (hereafter simply called the free energy density), can be written as $g_{\mathrm{s}}=\tau^{2-\alpha} \tilde{g}\left(p / \tau^{\beta \delta}\right){ }^{16}$ From the concept of two-scale-factor universality, we can extend the form to include the $N$ dependence. Putting the scale factors of $p$ and $\tau$ as $N^{f}$ and $N^{t}$, respectively, we may write

$$
g_{\mathrm{s}}(\tau, p) / T_{\mathrm{c}}=\left(N^{t} \tau\right)^{2-\alpha} \tilde{g}_{1}\left(N^{f} p^{*} /\left(N^{t} \tau\right)^{\beta \delta}\right)
$$

where $p^{*}$ is the non-dimensional osmotic pressure defined by $p^{*} \equiv p / T_{\mathrm{c}}$. This form of $g_{\mathrm{s}}$ enables us to deduce not only the scaling laws of the critical exponents but also the "scaling laws of the $N$ dependence of the critical amplitudes," namely the relations among the exponents defined by eq 12 . We can deduce the exponent-relations listed in Table I by eliminating $f$ and $t$. In the deduction, we should take care of the $N$ dependence of $T_{\mathrm{c}}$.

The law of eq 29 on Table I is derived as follows. From eq 16 with eq 17, we have,

$$
\Delta \phi \simeq \partial\left(g_{\mathrm{s}} / T_{\mathrm{c}}\right) / \partial p^{*}=N^{\beta t+f} \tau^{\beta} \tilde{g}_{1}{ }^{\prime}(x)
$$

where $x=N^{f} p^{*} /\left(N^{t} \tau\right)^{\beta \delta}$ and we have dropped the subscript 1 from $\Delta \phi_{1}$. Then, putting $x=0$ (or $p^{*}=0$ ) and comparing eq 18 with the definition of $b$ in eq 12 , we obtain, 


\section{K. ShinozaKi and T. Nose}

$$
b=\beta t+f
$$

Differentiating eq 18 with respect to $p^{*}, \chi$ is derived as,

$$
\chi T_{\mathrm{c}} \simeq-\left(1 / \phi_{\mathrm{c}}\right) \partial \Delta \phi / \partial p^{*}=N^{-\gamma t+2 f} \tau^{-\gamma} g_{1}{ }^{\prime \prime}(x)(20)
$$

Then, comparing eq 20 (with $x=0$ ) with the definitions of $g$ and $r$, we find,

$$
g+r+s=-\gamma t+2 f
$$

Similarly we have,

$$
C \simeq \partial^{2}\left(g_{\mathrm{s}} / T_{\mathrm{c}}\right) / \partial \tau^{2} \simeq N^{(2-\alpha) t} \tau^{-\alpha} \tilde{g}_{1}(x)
$$

and therefore,

$$
c=(2-\alpha) t=\mathrm{d} v t
$$

where $d$ is dimensionality of the space and we have used the scaling law $2-\alpha=\mathrm{d} v$. Eliminating $f$ and $t$ from eq 19, 21, and 22, we obtain eq 29.

The scaling law of eq 30 is derived as follows. We rewrite eq 17 in its equivalent form as,

$$
g_{\mathrm{s}}(\tau, p) / T_{\mathrm{c}}=\left(N^{f} p^{*}\right)^{1+\delta^{-1}} \tilde{g}_{2}\left(N^{t} \tau /\left(N^{f} p^{*}\right)^{1 / \beta \delta}\right)
$$

Differentiating eq 23 with respect to $p^{*}$ and putting $\tau=0$, we obtain,

$$
\left.\Delta \phi \simeq\left(1+\delta^{-1}\right) N^{\left(1+\delta^{-1}\right.}\right)^{f} p^{* \delta^{-1}} \tilde{g}_{2}(0)
$$

Therefore, comparing with the definition of $j$, we find

$$
j=-(1+\delta) f+s
$$

Eliminating $f$ and $t$ from eq 24, 19, and 21, we obtain eq 30 .

The scaling form of a correlation function $C(\tau, r)$ takes the form: ${ }^{16}$

$$
\begin{aligned}
C(\tau, r) & \equiv\langle\Delta \phi(0) \Delta \phi(r)\rangle \\
& =\left(N^{n \prime} r\right)^{-(d-2+\eta)} N^{2 f} \tilde{c}\left(N^{n \prime} r\left(N^{t} \tau\right)^{v}\right)
\end{aligned}
$$

where $n^{\prime}$ is the scale factor of the length. Only two of the exponents $f, t$, and $n^{\prime}$ are independent, which is proved as follows. From eq 25 , we find $\xi \propto N^{-n^{\prime-v} t}$ since $\tilde{c}$ should have the form $\tilde{c}(r / \xi)$. Therefore, $n=-n^{\prime}-v t$. The universal ratio connecting the correlation amplitudes with the thermodynamic ones, $R_{\xi} \equiv \xi_{0} A^{1 / d}$, leads to the relation,

$$
d n+c=0
$$

Therefore, from eq 26, 22, and the relation $n=-n^{\prime}-v t$, we find $n^{\prime}=0$, and then,

$$
n=-v t
$$

Thus, the correlation function has the form:

$$
C(\tau, r)=r^{-(d-2+\eta)} N^{2 f} \tilde{c}\left(r\left(N^{t} \tau\right)^{v}\right)
$$

The wave-vector-dependent osmotic compressibility $\chi(k)$ is proportional to the Fourier transform of $C(0, r)$; therefore, its $N$ dependence is the same as $C(0, r)$, i.e.,

$$
k=2 f
$$

Eliminating $f$ from eq 28 and 24, we obtain eq 31 .

The scaling law of eq 32 can be derived from eq 26 and 29.

Interfacial tension $\sigma$ is related to the free energy density $g_{\mathrm{s}}$ by, ${ }^{7}$

$$
\sigma \simeq \xi g_{\mathrm{s}}
$$

From eq 17 and 22, we find $g_{\mathrm{s}} \propto N^{c+s}$; therefore, with this relation, we obtain from the above equation

$$
m=n+c+s
$$

Inserting eq 26 into this equation, we obtain eq 33 .

\section{DISCUSSION}

The two-scale-factor universality is usually formulated with respect to the equation-of-state, ${ }^{7-9}$ and not to the free energy density as in the last section. As far as the $N$ dependence is concerned, equivalent results can be deduced from our formulation. In fact, the relations listed in Table I can also be derived from the universal ratios with a dimensional consideration, which shows the equivalence between the two formulations. Our formulation is convenient in a binary mixture since an equation-of-state for a mixture near the critical temperature is unfamilliar.

The scaling laws of $N$ dependence parallel the scaling laws of the critical exponents except for subtle modifications steming from the $N$ depen-

Table I. Scaling laws of $N$ dependence of critical amplitudes in polymer solutions

(Corresponding universal ratios are also shown.)

$\begin{array}{ll}R_{\mathrm{c}} \equiv A B^{-2} \Gamma & g+r-2 b+c+s=\theta \\ R \equiv \Gamma D B^{\delta-1} & g+r+j+b(\delta-1)=0 \\ R_{\mathrm{D}} \equiv D^{(\delta+1) / 2} D & (1+\delta) k / 2+j-s=0 \\ S \equiv B^{2} \Gamma^{-1} \xi_{0}^{d} & g+r-2 b+s=d n \\ \sigma \simeq \xi g_{\mathrm{s}} & m=-(d-1) n+s\end{array}$


dences of $\phi_{\mathrm{c}}$ and $T_{\mathrm{c}}$. We list below other useful relations equivalent to those listed in Table I:

$$
\begin{array}{rlrl}
R_{\xi} & \equiv \xi_{0} A^{1 / d} & c=-n d \\
T & \equiv B^{2} \Gamma^{-1} \xi_{0} \sigma_{0}^{-1} & & m-n=-(g+r)+s \\
R_{\mathrm{x}} & \equiv \Gamma D B^{\delta-1} & & j-c=-(\delta+1) b
\end{array}
$$

The classical values of the exponents derived in the first section $(c=k=r=-1 / 2, g=j=1 / 2, b=m=$ $-1 / 4, n=1 / 4$, and $s=0$ ) satisfy the scaling laws of eq $29,30,34$, and 35 , but not those of eq $26,31,32$, and 33. The breakdown of the latter corresponds exactly to the fact that the classical critical exponents do not satisfy Josephson's scaling law. This is due to the inconsistency in the derivation of classical values. ${ }^{11}$ The correlation function was derived neglecting the quartic term (Gaussian approximation) while the other functions were derived neglecting the gradient term (see eq 8 ). The classical critical exponents also do not satisfy the scaling laws corresponding to eq 31 , etc. but the real (nonclassical) values satisfy all the scaling laws. Therefore, we may expect that the real exponent of $N$ will satisfy all the scaling laws of the $N$ dependence.

De Gennes proposed, in his earlier work, ${ }^{17} \mathrm{a}$ scaling form of free energy for a polymer-solvent system similar to eq 17 . He also recently derived ${ }^{18}$ on essentially the same basis as the earlier one that,

$$
\begin{gathered}
\xi=R_{0}\left\{\left(\Theta-T_{\mathrm{c}}\right) /\left(T-T_{\mathrm{c}}\right)\right\}^{v} \\
\Delta \phi=N^{-1 / 2+\beta / 2}\left(\chi-\chi_{\mathrm{c}}\right)^{\beta}
\end{gathered}
$$

where $R_{0}$ is the unperturbed chain dimension and $\Theta$ the Flory temperature and $\chi$ the polymer-solvent interaction parameter. The above equations give

$$
n=1 / 2-v / 2 \quad \text { and } \quad b=-1 / 2+\beta / 2
$$

His treatment corresponds to the case when the two scale factors do not depend on the critical exponents; however, as seen from the renormalization-group technique of calculation, ${ }^{9}$ the scale factors depend on them generally. The present formulation results directly from the universality of critical phenomena and is applicable not only to a polymer-solvent system but also to a polymer-polymer and a polymer-polymer-solvent (pseudo-two-component) system.

At this stage, we can test some of the scaling laws with the experimental values: $n=0.28, g=0.48,{ }^{19}$ $r=-0.40, b=-0.34$, and $m=-0.44 .^{6}$ Thus, the left-hand-side of eq 32 is 0.76 and the r.h.s. is 0.84 ; the 1.h.s. of eq 33 is -0.44 and the r.h.s. -0.56 ; and the I.h.s. of eq 34 is 0.72 and the r.h.s. is 0.76 . Thus, we may conclude that the laws are approximately satisfied by the experimental data.

It should be remarked that the scaling laws of the $N$ dependence are the form of the universality characteristic in polymer solutions. The values of the exponents on $N$ have the same significance as the critical exponents. In regard to polymer solutions, further experimental study on critical amplitudes in the region of sufficiently high molecular weight are expected.

\section{REFERENCES}

1. P. Debye, J. Chem. Phys., 31, 680 (1959).

2. N. Kuwahara, D. V. Fenby, M. Tamsky, and B. Chu, J. Chem. Phys., 55, 1140 (1971).

3. M. Nakata, N. Kuwahara, and M. Kaneko, J. Chem. Phys., 62, 4278 (1975).

4. Q. H. Lao, B. Chu, and N. Kuwahara, J. Chem. Phys., 62, 2039 (1975).

5. T. Dobashi, M. Nakata, and Kaneko, J. Chem. Phys., 72, 6685, 6692 (1980).

6. K. Shinozaki, T. V. Tan, Y. Saito, and T. Nose, Polymer, to be published.

7. D. Stauffer, M. Ferer, and Michael Wortis, Phys. Rev. Lett., 29, 345 (1972).

8. A. Aharony and P. C. Hohenberg, Phys. Rev., B13, 3081 (1976).

9. C. Bervillier, Phys. Rev., B14, 4964 (1976).

10. K. Shinozaki and T. Nose, J. Phys. Soc. Jpn. Lett., 50, 1817 (1981).

11. P. Pfeuty and G. Toulouse, "Introduction to the Renormalization Group and to Critical Phenomena," John Wiley \& Sons, New York, 1977, Chapter 2.

12. A. Vrij and M. W. J. Van Den Esker, J. Chem. Soc., Faraday Trans. 2, 68, 513 (1972).

13. T. Nose, Polym. J., 8, 96 (1975).

14. A. Vrij and G. J. Roeberson, J. Polym. Sci., Polym. Phys. Ed., 15, 109 (1977).

15. B. Chu, Phys. Lett., 28A, 654 (1969).

16. H. E. Stanley, "Introduction to Phase Transitions and Critical Phenomena," Oxford University Press, Oxford, 1971.

17. P. G. De Gennes, Phys. Lett., 26A, 313 (1968).

18. P. G. De Gennes, "Scaling Concepts in Polymer Physics," Cornell University Press, Ithaca, N.Y., 1979, pp 121, 213.

19. K. Shinozaki, T. Hamada, and T. Nose, J. Chem. Phys., to be published. 